Check for updates

Cite this: RSC Adv., 2019, 9, 11842

\title{
Grape seed proanthocyanidin extract alleviates high-fat diet induced testicular toxicity in rats
}

\author{
Er Hui Wang, (D) *ab Zeng Li Yu, ${ }^{a}$ Yong Jun Bu, ${ }^{a}$ Peng Wei $\mathrm{Xu}{ }^{\mathrm{a}}$ Jin Yan $\mathrm{Xi}^{\mathrm{a}}$ \\ and Hai Yan Liang ${ }^{a}$
}

The present study aimed to investigate the protective effects of grape seed proanthocyanidin extract (GSPE) on high-fat diet (HFD) induced testicular damage, oxidative stress, and apoptotic germ cell death. Male rats ( $n=40$ ) were randomly divided into four groups: the control group (treated with physiological saline), HFD group, HFD + GSPE (100 mg kg ${ }^{-1}$ ) group and HFD + GSPE (300 $\mathrm{mg} \mathrm{kg}^{-1}$ ) group. Compared with the HFD group the rats of the GSPE-treated group showed improved serum testosterone levels, sperm quality and histological appearance of the testis tissue. Significant elevation of antioxidant enzyme (SOD, GSH, and GSH-Px) activities and remarkable reduction in MDA were also observed by GSPE administration, indicating that GSPE can decrease testicular oxidative stress. Finally, a significant reduction in spermatogenic cell apoptosis was detected by TUNEL assay. In summary, these results indicated that GSPE can suppress testicular dysfunction and this effect may be attributed to its antioxidant and antiapoptotic properties. The current study indicates that GSPE can be considered a promising candidate for use as a drug or a food supplement to alleviate HFD-induced testicular dysfunction.

Received 8th February 2019

Accepted 9th April 2019

DOI: 10.1039/c9ra01017c

rsc.li/rsc-advances antioxidants against obesity induced male reproductive function damage. ${ }^{\mathbf{8} 19}$ With regard to the undesirable consequences of obesity on male reproductive system, finding efficient protective pharmacological agents are of great therapeutic interest.

Several previous studies indicated that excessive germ cell apoptosis in the testes is a key pathophysiological process in the obesity-induced male spermatogenesis dysfunction. ${ }^{\mathbf{1 0 - 1 4}}$ Apoptosis is essential for cellular homeostasis and normal male germ cell development process. ${ }^{20,21}$ However, excessive testicular germ cell apoptosis, in turn, may promote the progression of infertility. Conversely, inhibiting excessive apoptosis attenuates HFD induced impairment of spermatogenesis. ${ }^{\mathbf{1 0 - 1 3}}$

Grape seed proanthocyanidin extracts (GSPE) are a group of biologically active polyphenols bioflavonoids diverse in chemical structure, pharmacology and characteristics. The protective effects of GSPE on the reproductive system have been demonstrated for various conditions. ${ }^{22-27}$ All these studies attributes the protective effects to its antioxidant and anti-apoptotic activities. Many papers published in recent decades have documented that, the putative beneficial effects of polyphenols are frequently related to their antioxidant activity. ${ }^{28}$ There in all these implying beneficial role of GSPE.

On the basis of the studies above mentioned, it is not difficult to realize that oxidative stress and apoptosis are the major players in HFD induced testicular dysfunction, although the mechanisms responsible for this remain unclear. In addition, the protective effect of GSPE on obesity induced impaired spermatogenesis has not yet been reported. Considering the anti-apoptosis and anti-oxidative properties of GSPE, the aim of
${ }^{a}$ Department of Nutrition and Food Hygiene, College of Public Health, Xinxiang Medical University, Xinxiang 453003, China.E-mail:weh1985@126.com

${ }^{b}$ Henan Collaborative Innovation Center of Molecular Diagnosis and Laboratory Medicine, Xinxiang Medical University, Xinxiang 453003, China 
the present study was to investigate potential protective effects of GSPE on HFD induced testicular apoptosis and oxidative damage in rats.

\section{Materials and methods}

\subsection{Chemicals}

GSPE (purity is more than $95 \%$ as analysed by UV, in which dimer is $56 \%$, trimer is $12 \%$, tetramer is $6.6 \%$ and monomer and other high-molecular mass oligomers is $20.4 \%$ as analysed by HPLC) was purchased from Tianjin Peak Natural Product Research Development Co., Ltd. Reduced glutathione (GSH) assay kit (Spectrophotometric method, cat. no. A006-1), glutathione peroxidase (GSH-PX) assay kit (Colorimetric method, cat. no. A005), superoxide dismutase assay kit (Hydroxylamine method, cat. no. A001-1-1), and malondialdehyde (MDA) assay kit (TBA method, cat. no. A003-1) were obtained from Nanjing Jian Cheng Bioengineering Institute (Nanjing, China). TUNEL assay kit was obtained from Promega Corp. (cat. no. G7360). Sex hormone specific kits purchased from Beijing North Institute of Biological Technology.

\subsection{Animals and experimental design}

All of the animals received humane care according to the criteria outlined in the Guide for the Care and Use of the Animal Management Rules of the Health Ministry of the People's Republic of China (documentation number 55, 2001, China) and were approved by the Animal Care and Use Committees of Xinxiang Medical University. A total of 40 male Sprague Dawley rats (90-120 g, 4 weeks) were quarantined 7 days and then randomly divided into four groups: control group (standard diet), HFD group, HFD + GSPE (100 $\left.\mathrm{mg} \mathrm{kg}^{-1}\right)$ and HFD + GSPE $\left(300 \mathrm{mg} \mathrm{kg}^{-1}\right.$ ) group. Rats in the control group were fed with a normal diet (control, $10 \mathrm{kcal} \%$ fat, D12450B, Research Diets), whereas the other rats were fed with an (HFD, $45 \mathrm{kcal} \%$ fat, D12451, Research Diets, New Brunswick, NJ, USA) for 13 weeks to induce obesity (Table 1). Rats in the HFD + GSPE group were orally administered with $100 \mathrm{mg} \mathrm{kg}^{-1}$ GSPE (G100 group) or $300 \mathrm{mg} \mathrm{kg}^{-1}$ GSPE (G300 group) once per day for 13 weeks, and the other two groups received the same volume of vehicle as control. The dose of GSPE used in this study was selected on the basis of the previous studies. ${ }^{22-27}$ At the end of the experiment, the rats were sacrificed under sodium pentobarbital anesthesia (60 $\mathrm{mg} \mathrm{kg} \mathrm{kg}^{-1}$, ip). The testes and epididymis were quickly removed, cleared of adhering connective tissue, weighed and assayed immediately. One of the testes was fixed in neutralformalin solution for histopathological examinations. The other testis samples were also stored at $-80{ }^{\circ} \mathrm{C}$ until biochemical analyses.

\subsection{Serum hormone analysis}

On the last day of the study blood samples were collected and immediately placed in refrigerator stored at $4{ }^{\circ} \mathrm{C}$ until centrifuged at $3000 \mathrm{rpm}$ for 10 minutes to separate serum. Serum was collected and stored at $-20{ }^{\circ} \mathrm{C}$ until analysis. Serum levels of follicle-stimulating hormone (FSH), luteotropic hormone (LH) and testosterone (T) were measured using hormone specific kits purchased from Beijing North Institute of Biological Technology by radioimmunoassay (RIA) method.

\subsection{Epididymis sperm concentration and motility}

The head of left epididymitis was placed in clean $30 \mathrm{~mm}$ dishes containing with $4 \mathrm{~mL}$ pre-warmed M199 and minced with a fine scissors to allow spermatozoa to swim out for 10 to $15 \mathrm{~min}$ at $37^{\circ} \mathrm{C}$. After incubation, the epididymal tissue-fluid mixture was filtered to separate the supernatant from tissue particles. The sperm suspensions were evaluated for sperm head counts, viability, motility and morphology following the procedure of as described below. The sperm head counts were determined with a hemocytometer. A sample of $0.5 \mathrm{~mL}$ of the sperm suspension was diluted with $9.5 \mathrm{~mL}$ of physiological saline. Approximately $10 \mu \mathrm{L}$ of diluted sperm suspension was transferred to each counting chamber and then was allowed to stand for $5 \mathrm{~min}$ and counted under a light microscope at $400 \times$ magnification. ${ }^{29}$ The total sperm counts $\left(\times 10^{6} / \mathrm{ml}\right)$ were calculated. The determination of sperm viability and motility was carried out according to the procedure described by previous article. ${ }^{18}$ Sperm morphological examination was performed as described by Terpsidis et al. with slight modification (a drop of the sperm suspension was spread onto a new glass plate for each sample and was allowed to dry in air). ${ }^{30}$

\subsection{Determination of testicular oxidative stress markers}

Testes tissues were taken from the deep-freezer and weighed. They were then transferred to cold glass tubes and diluted with a nine-fold volume of phosphate buffer (pH 7.4). For the enzymatic analysis, testicular tissues were minced and homogenized using an ultrasonic cell breaker. The homogenates were centrifuged at $3000 \mathrm{rpm}$ for $10 \mathrm{~min}$ at $4{ }^{\circ} \mathrm{C}$. The supernatant was used for the assay of GSH, GSH-Px, SOD, and MDA based on the methodology of assay kits.

\subsection{Histopathological examination}

A complete gross visual pathology inspection was conducted on testes of all animals during the necropsy by a senior pathologist. The testes were trimmed of extraneous fat and weighed immediately. The ratios of testes weight to terminal body weights were calculated. Tissue sections from testes were fixed with $4 \%$ formalin for $24 \mathrm{~h}$, embedded in paraffin, sectioned to $5 \mu \mathrm{m}$ and stained in hematoxylin and eosin for microscopic observation.

\subsection{TUNEL assay}

Testicular cell apoptosis was measured using a TUNEL assay kit (cat. no. G7360; Promega) according to the manufacturer's protocol. To assess testicular cells apoptosis in the testes, 200 different seminiferous tubules were randomly selected in each group (20 tubules per rat) and observed at a magnification of $\times 400$. To find out the apoptotic germ cell index (AI), the percent of labeled germ cells was calculated in 20 tubules for each rat and finally, apoptotic germ cell index-1 (AI-1) as the number of tubules containing at least one TUNEL-positive cells per 100 
Table 1 Mean weekly body weights among groups (mean \pm SD, $n=10$ )

\begin{tabular}{|c|c|c|c|c|}
\hline Week & Control & HFD & HFD + GSPE $\left(100 \mathrm{mg} \mathrm{kg}^{-1}\right)$ & HFD + GSPE $\left(300 \mathrm{mg} \mathrm{kg}^{-1}\right)$ \\
\hline 0 & $129.94 \pm 4.77$ & $135.00 \pm 5.94$ & $134.60 \pm 3.69$ & $128.50 \pm 16.61$ \\
\hline 1 & $191.30 \pm 10.10$ & $215.00 \pm 12.84$ & $212.80 \pm 7.93$ & $205.70 \pm 10.10$ \\
\hline 3 & $300.60 \pm 22.66$ & $358.30 \pm 32.46$ & $351.70 \pm 16.96$ & $343.00 \pm 21.53$ \\
\hline 4 & $339.60 \pm 27.75$ & $407.10 \pm 38.52$ & $402.80 \pm 21.89$ & $395.70 \pm 27.01$ \\
\hline 5 & $377.40 \pm 31.85$ & $455.10 \pm 44.70$ & $441.40 \pm 25.26$ & $426.62 \pm 42.34$ \\
\hline 8 & $459.10 \pm 39.53$ & $563.50 \pm 59.73$ & $543.00 \pm 35.90$ & $533.10 \pm 28.59$ \\
\hline 9 & $481.10 \pm 44.50$ & $593.60 \pm 58.35$ & $573.80 \pm 42.41$ & $564.00 \pm 31.16$ \\
\hline 10 & $503.20 \pm 47.00$ & $625.80 \pm 61.95$ & $598.30 \pm 48.49$ & $593.80 \pm 33.06$ \\
\hline 11 & $515.80 \pm 55.18$ & $646.30 \pm 59.13$ & $617.60 \pm 54.10$ & $605.50 \pm 33.76$ \\
\hline 12 & $526.50 \pm 58.57$ & $663.90 \pm 53.54$ & $622.00 \pm 59.13$ & $612.00 \pm 29.66$ \\
\hline 13 & $537.07 \pm 56.08$ & $697.10 \pm 39.55$ & $614.10 \pm 59.01$ & $604.00 \pm 27.46$ \\
\hline
\end{tabular}

tubules and apoptotic index-2 (AI-2) was described as the number of TUNE-positive cells per 100 tubules. $^{31,32}$

\subsection{Statistical analysis}

Data were presented as means \pm SD (standard deviation). Statistical analyses were performed by using one-way ANOVA test to evaluate the homogeneity of the data followed by a least squared differences model or Dunnett's multiple comparison test if the homogeneity evaluation indicated significant deviation variances in SPSS for windows version 11.5 (SPSS Inc., Chicago, IL, USA). Differences were considered to be significant at $p<0.05$.
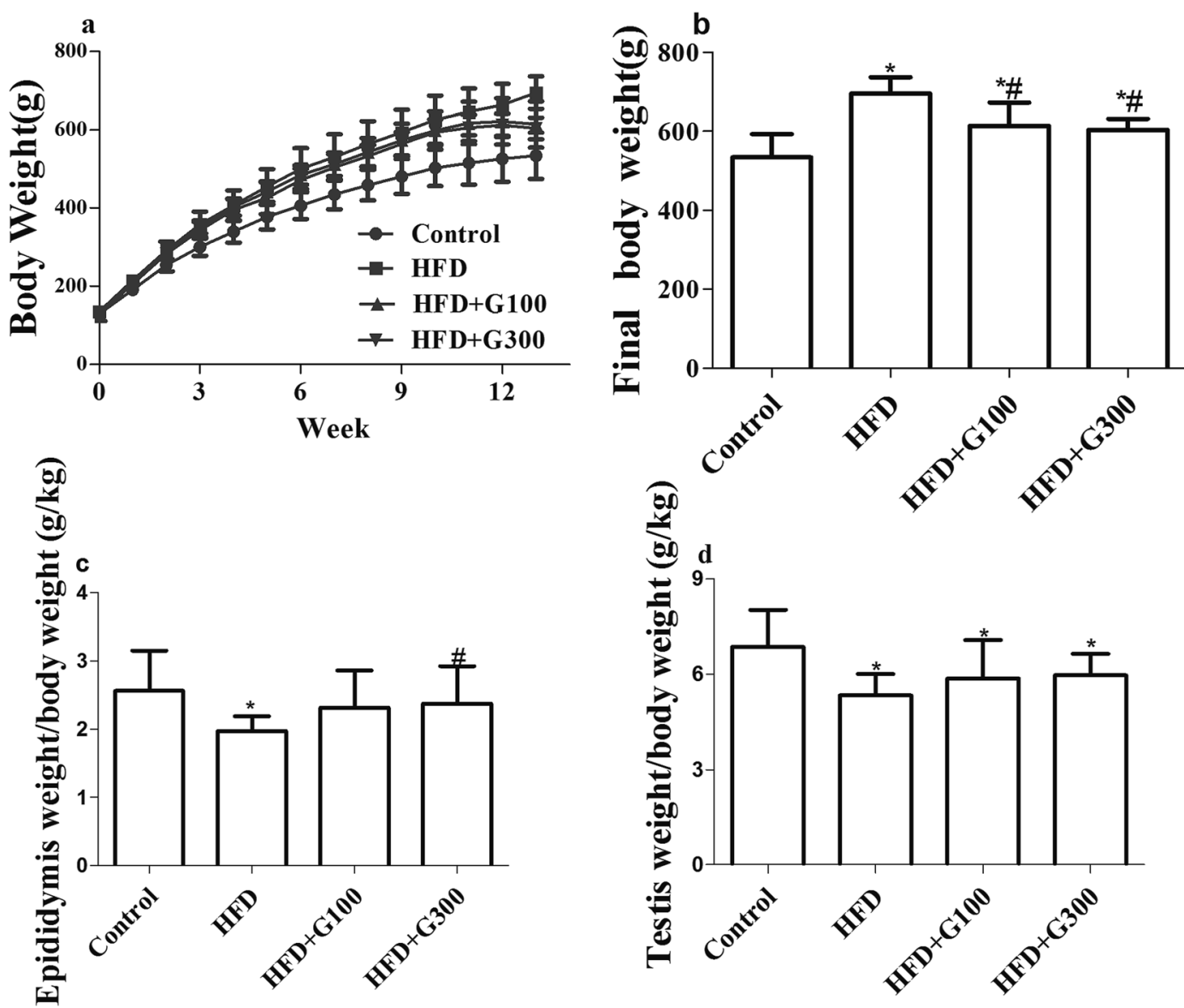

Fig. 1 HFD treatment significantly increased the body weights gain ( $a$ and b) and reduced reproductive organ weight/body weight ratios (c and d) of rats compared with the control group. GSPE treatment alleviated this tendency. ${ }^{*} P<0.05$ vs. control and $\# P<0.05$ vs. HFD. 

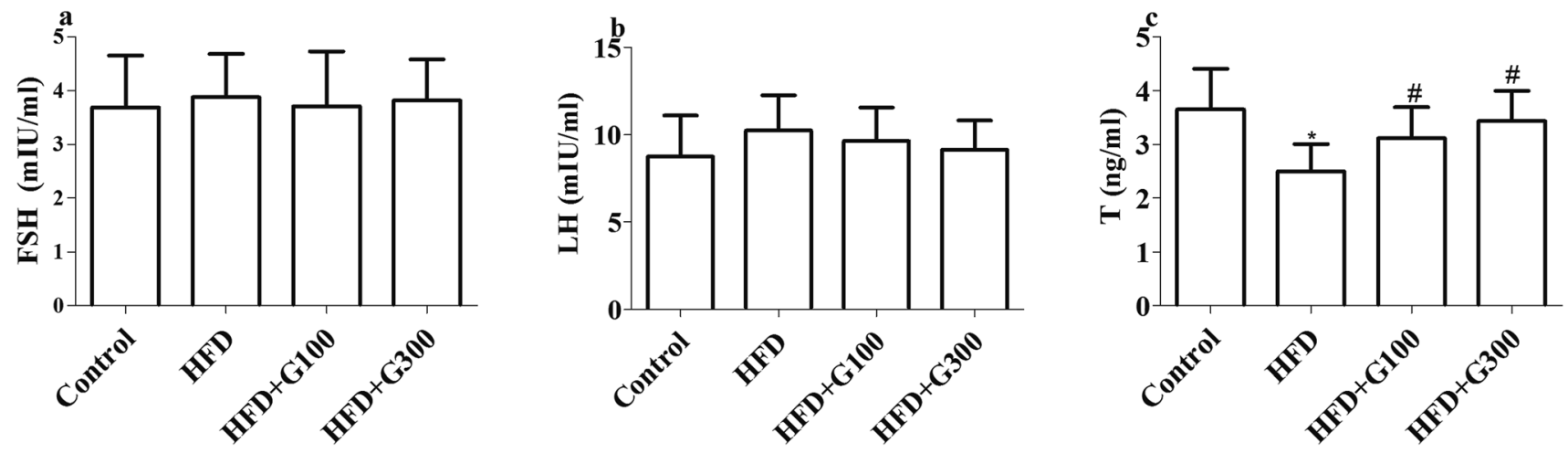

Fig. 2 HFD feeding significantly decreased the serum T level of rats compared with the control group. GSPE supplementary restored HFD induced serum $T$ level decline. No significant differences were observed in serum level of FSH and LH among groups $* P<0.05$ vs. control and \#P $<0.05$ vs. HFD.

\section{Results}

\subsection{GSPE reduce body weights gain and organ/body weight} ratios decrease

After 13 weeks feeding, the body weight of rat in both the HFD and GSPE groups increased significantly compared with the control group $(P<0.05$; Fig. 1$)$. GSPE treatment significantly attenuated HFD induced body weight increases ( $P<0.05$; Fig. 1). GSPE treatment also showed a tendency of alleviate HFD induced organ weights loss of the testes and epididymitis, but there were no significant differences in weight loss of testes between GSPE treatment groups (100 $\mathrm{mg} \mathrm{kg}^{-1}$ and $300 \mathrm{mg} \mathrm{kg}^{-1}$ ) and the HFD group (Fig. 1). The GSPE (100 $\mathrm{mg} \mathrm{kg}^{-1}$ ) resulted in insignificant decrease in weight loss of epididymitis compared

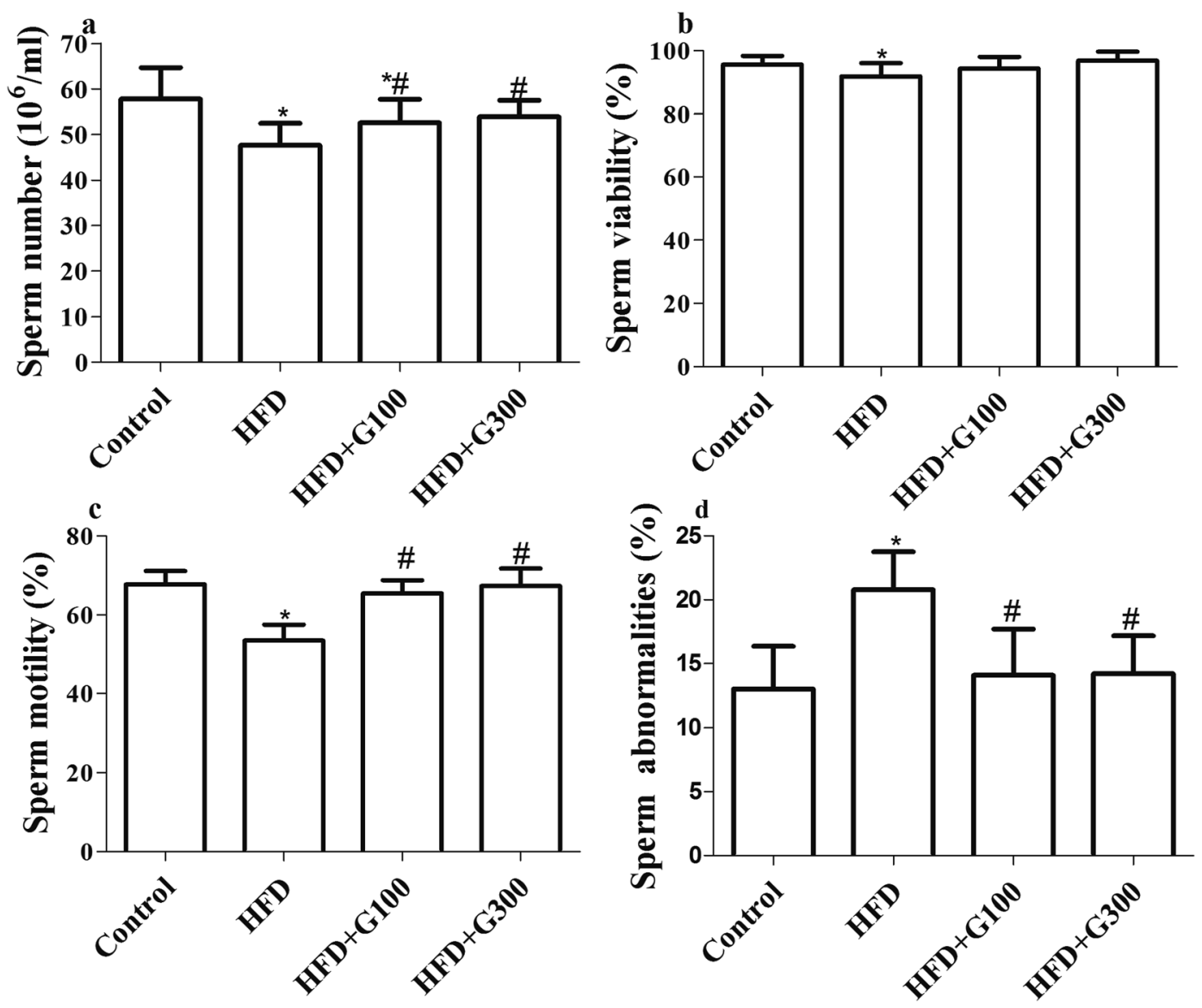

Fig. 3 Sperm parameters such as concentration, sperm viability and motility were significantly decreased and sperm abnormalities increased in HFD rats. GSPE treatment significantly attenuated HFD induced sperm parameters decline. $* P<0.05$ vs. control and \#P<0.05 vs. HFD. 

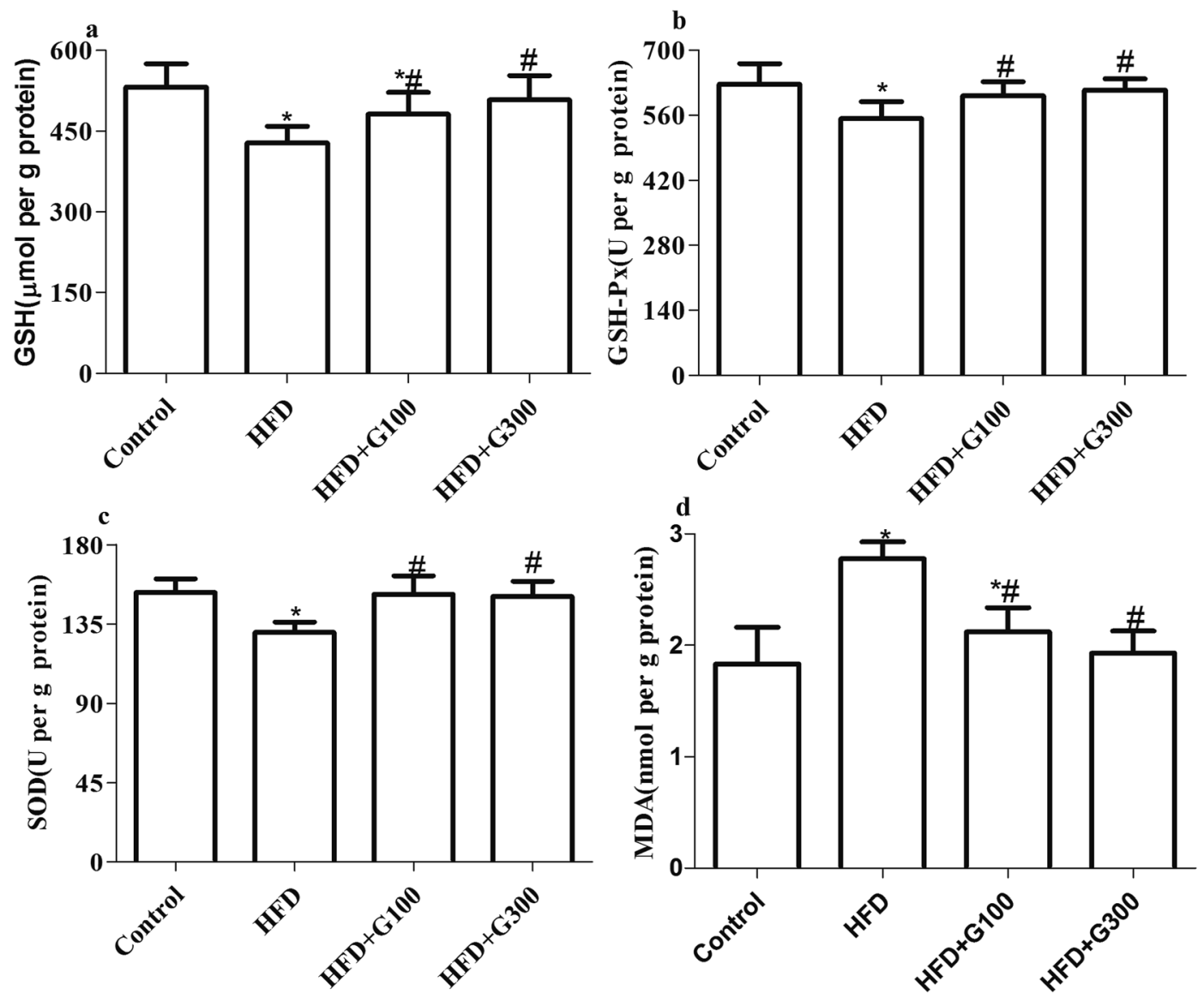

Fig. 4 Levels of GSH and MDA, and GSH-Px, SOD activities in the testes of rats were markedly decreased after HFD treatment. GSPE supplementary decrease HFD induced oxidative stress in the testes.

with the HFD group, while a significant difference was observed between the GSPE (300 $\left.\mathrm{mg} \mathrm{kg}^{-1}\right)$ treatment group and the HFD group $(P<0.05$; Fig. 1).

\subsection{GSPE attenuates HFD induced abnormal serum sexual hormone levels}

Rats fed with HFD presented significantly decreased serum $\mathrm{T}$ levels compared with the control group ( $P<0.05$; Fig. 2). However, GSPE treatment significantly ameliorated HFD induced abnormal serum T levels ( $P<0.05$; Fig. 2). However, there were no significant differences in serum level of FSH and LH among groups ( $P>0.05$; Fig. 2).

\subsection{GSPE attenuates HFD induced impaired spermatogenesis}

After HFD exposure for 13 weeks obvious impaired spermatogenesis effects were observed. Sperm parameters such as concentration, sperm viability and motility were significantly decreased while sperm abnormalities increased in rats subjected to a HFD diet $(P<0.05$; Fig. 3). GSPE treatment significantly attenuated HFD induced impaired spermatogenesis $(P<$ 0.05; Fig. 3).

\subsection{GSPE ameliorate HFD-induced testicular oxidative stress}

The MDA and GSH levels and GSH-Px and SOD activities of all the treatment groups are shown in Fig. 4. HFD administration caused significant $(P<0.05)$ increases in MDA levels, significant decreases in GSH levels $(P<0.05)$, GSH-Px $(P<0.05)$ and SOD activities $(P<0.05)$ of the testes tissue compared with the control group. However, the administration of GSPE $(100 \mathrm{mg}$ $\mathrm{kg}^{-1}$ and $400 \mathrm{mg} \mathrm{kg}^{-1}$ ) reverted MDA increase and prevented the decreases in these antioxidants above mentioned induced by HFD exposure (Fig. 4).

\subsection{GSPE improves pathological damage of testes induced by HFD}

Significantly decreased testes weight/body ratios were found in the HFD group compared with the control group $(P<0.05$; Fig. 1), which was also confirmed by testes histological changes analysis (Fig. 5). The testes of the control group showed a regular histological structure of the seminiferous tubules fulfilled with tight and organized spermatogenic cells at different stages. In the HFD treatment group, the seminiferous tubules structures were disrupted with disordered spermatogenic cells and more vacuoles. Notably, decreased spermatogenic cells and interstitial cells were also observed. 


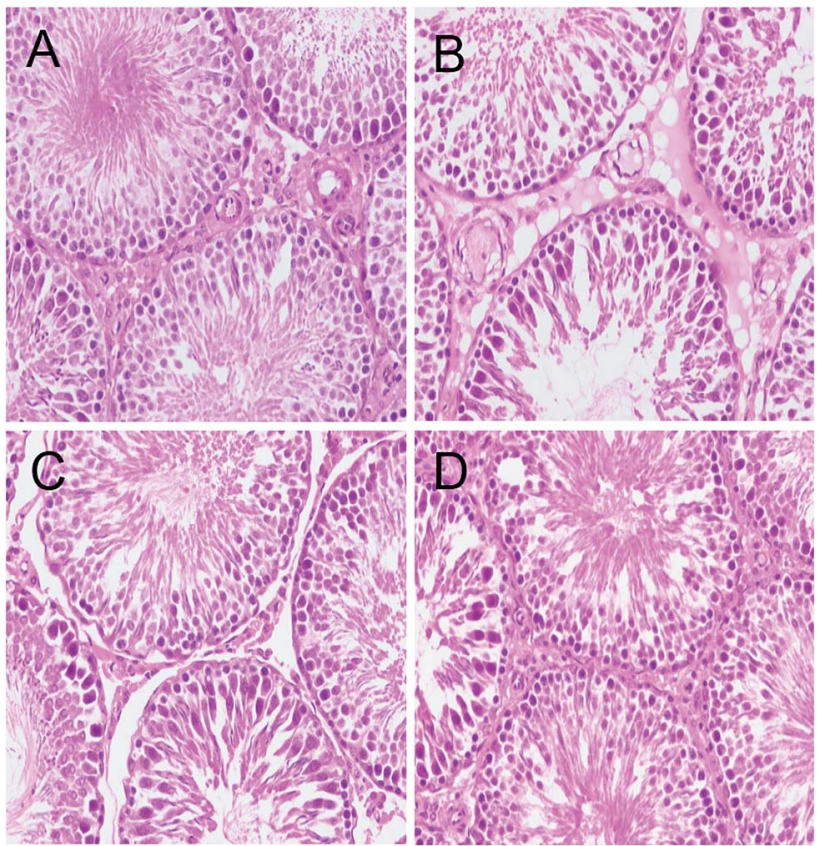

Fig. 5 Light microscopy of testes tissue in different groups. H\&E $\times 200$ : (A-D, Control group, HFD group, HFD + GSPE (100 $\left.\mathrm{mg} \mathrm{kg}^{-1}\right)$ group and HFD + GSPE (300 $\mathrm{mg} \mathrm{kg}^{-1}$ ) group). (A) In control group, normal testicular architecture was seen; (B) after HFD treatment, severe testes damage was noted; ( $C$ and $D)$ there was an improvement in the seminiferous tubule structure in GSPE-treated HFD rats.

Histopathology changes of the testicular illustrated that, compared with the control group, HFD feeding induced obvious pathological damage in the testicular tissue. While histological changes were improved with GSPE treatment (Fig. 5).

\subsection{GSPE suppresses HFD-induced apoptosis in testicular germ cells}

The results of TUNEL analysis revealed that rats in the HFD group had a significantly higher rate of apoptosis compared with the control rats, and that GSPE significantly inhibited HFD induced testicular cell apoptosis $(P<0.05$; Fig. 6$)$.

\section{Discussion}

The present study explored the protective effect of GSPE on HFD exposure induced oxidative damage and germ cell apoptosis in rats. We found that HFD could provoke oxidative stress and sperm damage in rats, and GSPE could repair oxidative damage and improve sperm quality to some extent. The results of the present study demonstrated that GSPE treatment ameliorate HFD induced serum $\mathrm{T}$ decline and testicular cell apoptosis. Additionally, GSPE treatment improves pathological damage of testes induced by HFD. Taken together, GSPE have protective effects in HFD-induced spermatogenesis dysfunction.

Sex hormones such as FSH, LH and T are known to play important roles in the spermatogenesis process. Especially, $\mathrm{T}$ synthesized in Leydig cells, plays key roles in the development of male reproductive tissues and spermatogenesis. Consistent with previous studies, ${ }^{9-13}$ in our experiment, the serum $\mathrm{T}$ levels were markedly decreased in the HFD group when compared with the control group. The present study demonstrated that GSPE up regulates the serum level of $\mathrm{T}$, which suggests that restored $\mathrm{T}$ levels may contribute to the protective effects of GSPE. However how testosterone biosynthesis pathway could be modulated by these extracts is still undiscovered. Future studies involving the expression level of testosterone biosynthesis pathway related genes and proteins evaluated in future studies may help to explore the mechanism. GSPE had no significant effect on FSH and LH levels, which may indicates that it does not affect pituitary hormones.

To evaluate the effects of GSPE on the spermatogenesis in rat testes fed with HFD, sperm quality was determined in the present study. The results showed that sperm quality was markedly elevated by GSPE administration in HFD rats, which may be due to the stimulated effects of GSPE on testicular testosterone, as high testosterone level is essential for seminiferous tubules, spermatogenesis and sperm maturation.

Oxidative stress, a state related to increased cellular damage triggered by oxygen and oxygen-derived free radicals known as reactive oxygen species (ROS), has been identified as one of the many mediators of male infertility by causing sperm dysfunction. During this process, augmented production of ROS overwhelms the body's antioxidant defences. ${ }^{33}$ Excessive ROS result in severe oxidative damage to not only stimulate consecutive reactions resulting in further free radicals production, but also induce lipid peroxidation that lead to producing lipid peroxide (LPO) such as MDA, which then results in the damage of cellular compartments and function. Antioxidant enzymes, such as SOD, GSH, and GSH-Px play vital roles in the elimination of ROS, which defend cellular integrity against free radicalinduced damages. Oxidative stress has been shown in this study and was in agreement with those reported in other studies. ${ }^{9,34}$ In the present study, exposure to HFD induce an increase in the production of MDA and decrease in GSH level and activities of SOD and GSH-Px, which may be due to the overproduction of ROS and the deficiency of antioxidant defenses, thereby induced testicular oxidative damage. However, the increased MDA production and decreased in GSH level, activities of SOD and GSH-Px were significantly restored by GSPE treatment, suggesting that GSPE attenuated HFD-induced oxidative damage in the testes of rat.

Many previous studies have proved the fact that HFD exposure can result in testicular histopathological lesions and decreased reproductive organ weights..$^{8-14}$ In the present study, HFD-induced testicular damage was supported by the decrease in relative weights of tests, sperm parameters, low levels of serum $\mathrm{T}$, which may be consequences of elevated oxidative stress and excessive apoptosis in the testes. However, the adverse alterations induced by HFD exposure were alleviated by GSPE treatment.

Oxidative stress in the testes has already been considered as potent inducers of cell apoptosis. ${ }^{35}$ Many studies have been revealed that excessive apoptosis of germ cell plays a key role in male reproductive dysfunction induced by HFD. ${ }^{9-14}$ 

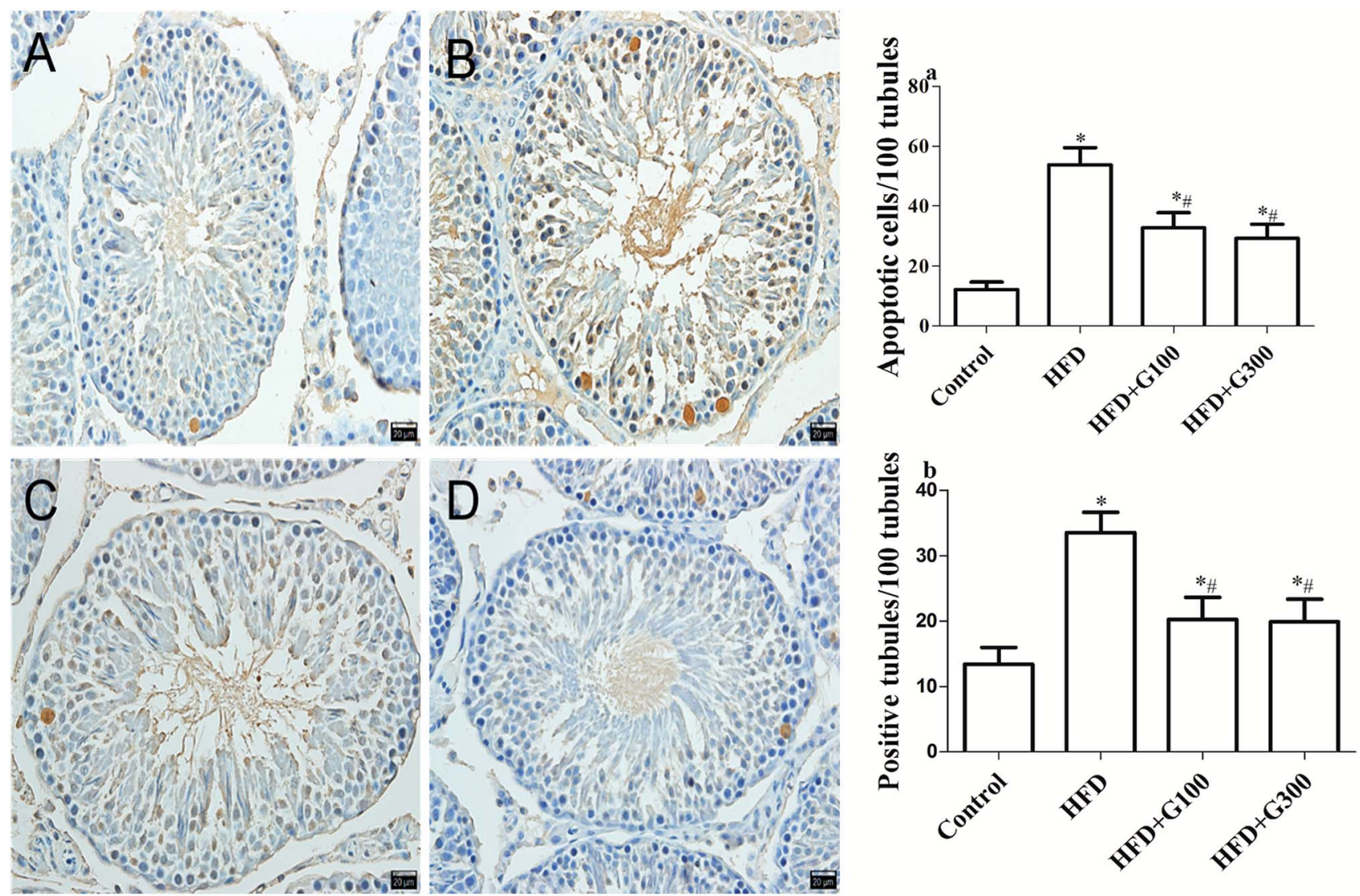

Fig. 6 TUNEL: representative photographs of TUNEL staining among groups ((A-D) control group, HFD group, HFD + GSPE (100 mg kg $\left.{ }^{-1}\right)$ group and HFD + GSPE (300 $\mathrm{mg} \mathrm{kg}^{-1}$ ) group). Positive cells of TUNEL staining were increased in HFD rats. Treatment with GSPE markedly reduced the reactivity and the number of germ cell apoptosis $(n=10)$. $* P<0.05$ vs. control and $\# P<0.05$ vs. HFD.

Spermatozoa production in the testes is a regulated balance between germ cell proliferation and germ cell apoptosis. ${ }^{36}$ Inhibiting excessive cell apoptosis were proved to be an effective treatment for defective spermatogenesis induced by HFD. ${ }^{9-13}$ The low concentration of spermatozoa, poor morphology after HFD feeding appeared to be correlated with pathological damage of the testes and high levels of testicular cell apoptosis. Our data simply revealed that GSPE treatment significantly inhibits testicular cell apoptosis, which suggests that apoptosis may be one of the underlying mechanisms by which GSPE protects against HFD induced defective spermatogenesis.

In conclusion, the present study suggested that HFD caused severe testicular oxidative damage and apoptosis. GSPE treatment can attenuate HFD induced testicular toxicity by decreasing oxidative damage and testicular apoptosis. These data provide important insights into the application of GSPE in alleviating HFD-induced testicular dysfunction, GSPE may be a potential novel therapeutic medicine for obesity associated male reproductive dysfunction.

\section{Conflicts of interest}

The authors declare that there are no conflicts to declare.

\section{Acknowledgements}

The authors thank grants from the National Natural Science Foundation of China (Grant No. 81703230) and the Key Scientific Research Project of Universities in Henan Province (Grant No. 17A330005) for financially support. We also thank pathologist lin Gao of Wuhan Biofavor Biotechnology services Co., Ltd for her excellent histopathological examination work.

\section{References}

1 F. Franceschi, R. Papalia, M. Paciotti, E. Franceschetti, A. Di Martino, N. Maffulli and V. Denaro, Obesity as a Risk Factor for Tendinopathy: A Systematic Review, Int. J. Endocrinol., 2014, 2014, 1-10.

$2 \mathrm{~K}$. Castetbon, Trends in adult body-mass index in 200 countries from 1975 to 2014: a pooled analysis of 1698 population-based measurement studies with 19.2 million participants, Lancet, 2016, 387, 1377-1396.

3 Y. Liu and Z. Ding, Obesity, a serious etiologic factor for male subfertility in modern society, Reproduction, 2017, 154, R123-R131. 
4 J. R. Craig, T. G. Jenkins, D. T. Carrell and J. M. Hotaling, Obesity, male infertility, and the sperm epigenome, Fertil. Steril., 2017, 107, 848-859.

5 N. O. Palmer, H. W. Bakos, T. Fallston and M. Lane, Impact of obesity on male fertility, sperm function and molecular composition, Spermatogenesis, 2012, 2, 253-263.

6 P. Gharagozloo and R. J. Aitken, The role of sperm oxidative stress in male infertility and the significance of oral antioxidant therapy, Hum. Reprod., 2011, 26, 1628-1640.

7 C. Wright, S. Milne and H. Leeson, Sperm DNA damage caused by oxidative stress: modifiable clinical, lifestyle and nutritional factors in male infertility, Reprod. Biomed. Online, 2014, 28, 684-703.

8 S. Gujjala, M. Putakala, V. Gangarapu, S. Nukala, R. Bellamkonda, R. Ramaswamy and S. Desireddy, Protective effect of Caralluma fimbriata against high-fat diet induced testicular oxidative stress in rats, Biomed. Pharmacother., 2016, 83, 167-176.

9 F. Erdemir, D. Atilgan, F. Markoc, O. Boztepe, B. SuhaParlaktas and S. Sahin, The effect of diet induced obesity on testicular tissue and serum oxidative stress parameters, Actas Urol. Esp., 2012, 36, 153-159.

10 W. J. Yan, Y. Mu, N. Yu, T. L. Yi, Y. Zhang, X. L. Pang, D. Cheng and J. Yang, Protective effects of metformin on reproductive function in obese male rats induced by highfat diet, J. Assist. Reprod. Genet., 2015, 32, 1097-1104.

11 M. Yang, W. J. Yan, T. L. Yin and J. Yang, Curcumin ameliorates high-fat diet-induced spermatogenesis dysfunction, Mol. Med. Rep., 2016, 14, 3588-3594.

12 H. Wang, Y. Z. Cai, Y. Shao, X. F. Zhang, N. Li, H. Y. Zhang and Z. G. Liu, Fish Oil Ameliorates High-Fat Diet Induced Male Mouse Reproductive Dysfunction via Modifying the Rhythmic Expression of Testosterone Synthesis Related Genes, Int. J. Mol. Sci., 2018, 19, 1325.

13 X. L. Miao, G. M. Gao, L. Jiang, R. Xu and D. P. Wan, Asiatic acid attenuates high-fat diet-induced impaired spermatogenesis, Exp. Ther. Med., 2018, 15, 2397-2403.

14 S. Ghosh and S. Mukherjee, Testicular germ cell apoptosis and sperm defects in mice upon long-term high fat diet feeding, J. Cell. Physiol., 2018, 233, 6896-6909.

15 W. Renata, W. J. Karol and S. Jolanta, The role of oxidative stress and antioxidants in male fertility, Cent. Eur. J. Urol., 2013, 66, 60-67.

16 S. Bisht, M. Faiq, M. Tolahunase and R. Dada, Oxidative stress and male infertility, Nat. Rev. Urol., 2017, 14, 470-485.

17 P. Gharagozloo and R. J. Aitken, The role of sperm oxidative stress in male infertility and the significance of oral antioxidant therapy, Hum. Reprod., 2011, 26, 1628-1640.

18 X. L. Chen, L. Z. Gong and J. X. Xu, Antioxidative activity and protective effect of probiotics against high-fat diet-induced sperm damage in rats, Animal, 2013, 7, 287-292.

19 R. A. Mohammadi, I. Salehi and M. Mortazavi, Protective effects of restricted diet and antioxidants on testis tissue in rats fed with high-fat diet, Iran. Biomed. J., 2015, 19, 96101.

20 L. D. Russell, H. Chiarini-Garcia, S. J. Korsmeyer and C. M. Knudson, Bax-dependent spermatogonia apoptosis is required for testicular development and spermatogenesis, Biol. Reprod., 2002, 66, 950-958.

21 C. E. Schaller, C. L. Wang, G. Beck-Engeser, L. Goss, H. S. Scott, M. S. Anderson and M. Wabl, Expression of Aire and the early wave of apoptosis in spermatogenesis, $J$. Immunol., 2008, 180, 1338-1343.

22 Y. M. Zhao, L. P. Gao, H. L. Zhang, J. X. Guo and P. P. Guo, Grape seed proanthocyanidin extract prevents DDP-induced testicular toxicity in rats, Food Funct., 2014, 5, 605-611.

23 S. G. Li, Y. S. Ding, Q. Niu, S. Z. Xu, L. J. Pang, R. L. Ma, M. X. Jing, G. L. Feng, J. M. Liu and S. X. Guo, Grape Seed Proanthocyanidin Extract Alleviates Arsenic-induced Oxidative Reproductive Toxicity in Male Mice, Biomed. Environ. Sci., 2015, 28, 272-280.

24 Y. Wang, F. Chen, M. Liang, S. Chen, Y. Zhu, Z. Zou and B. Shi, Grape seed proanthocyanidin extract attenuates varicocele induced testicular oxidative injury in rats by activating the Nrf2 antioxidant system, Mol. Med. Rep., 2018, 17, 1799-1806.

25 M. F. Sönmez and S. Tascioglu, Protective effects of grape seed extract on cadmium-induced testicular damage, apoptosis, and endothelial nitric oxide synthases expression in rats, Toxicol. Ind. Health, 2015, 32, 1486.

26 L. Su, Y. Deng, Y. Zhang, C. Li, R. Zhang, Y. Sun, K. Zhang, J. Li and S. Yao, Protective effects of grape seed procyanidin extract against nickel sulfate-induced apoptosis and oxidative stress in rat testes, Toxicol. Mech. Methods, 2011, 21, 487-494.

27 Y. Zhao, H. Zhang and L. Gao, Anti-apoptotic Effect of Grape Seed Proanthocyanidin Extract on Cisplatin-induced Apoptosis in Rat Testis, Food Sci. Technol. Res., 2015, 21, 805-811.

28 T. R. Anna, R. M. Lamuela-Raventos and J. J. Moreno, Polyphenols, food and pharma. Current knowledge and directions for future research, Biochem. Pharmacol., 2018, 156, 186-195.

29 S. Suresh, E. Prithiviraj and S. Prakash, Effect of Mucuna pruriens on oxidative stress mediated damage in aged rat sperm, Int. J. Androl., 2010, 33, 22-32.

30 K. I. Terpsidis, M. G. Papazahariadou, I. A. Taitzoglou, N. G. Papaioannou, M. P. Georgiadis and I. T. Theodoridis, Toxoplasma gondii: reproductive parameters in experimentally infected male rats, Exp. Parasitol., 2009, 121, 238-241.

31 S. Shokri, R. J. Aitken, M. Abdolvahhabi, F. Abolhasani, F. M. Ghasemi, I. Kashani, S. Ejtemaeimehr, S. Ahmadian, B. Minaei, M. A. Naraghi and M. Barbarestani, Exercise and supraphysiological dose of nandrolone decanoate increase apoptosis in spermatogenic cells, Basic Clin. Pharmacol. Toxicol., 2010, 106, 324-330.

32 R. Keyhanmanesh, G. Hamidian, M. R. Alipour, M. Ranjbar and $\mathrm{H}$. Oghbaei, Protective effects of sodium nitrate against testicular apoptosis and spermatogenesis impairments in streptozotocin-induced diabetic male rats, Life Sci., 2018, 211, 63-73. 
33 A. Ashok, V. Gurpriya, O. Chloe and P. Stefan S du, Effect of Oxidative Stress on Male Reproduction, World J. Mens Health, 2014, 32, 1-17.

34 J. Zhao, L. L. Zhai, Z. Liu, S. Wu and L. P. Xu, Leptin Level and Oxidative Stress Contribute to Obesity-Induced Low Testosterone in Murine Testicular Tissue, Oxid. Med. Cell. Longevity, 2014, 2014, 190945.
35 J. Yang, X. Zong, G. Wu, S. Lin, Y. Feng and J. Hu, Taurine increases testicular function in aged rats by inhibiting oxidative stress and apoptosis, Amino Acids, 2015, 47, 1549-1558.

$36 \mathrm{H}$. A. Jeng and S. M. Bocca, Influence of exposure to benzo[a] pyrene on mice testicular germ cells during spermatogenesis, J. Toxicol., 2013, 2013, 387850. 